\title{
Three rules for technological fixes
}

\author{
Not all problems will yield to technology. Deciding which will and which won't should be central \\ to setting innovation policy, say Daniel Sarewitz and Richard Nelson.
}

or some social problems, scientific research and technological innovation deliver significant progress, whereas for others, such activities lead to little if any improvement. Remarkable advances

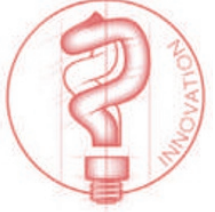

artefact, distinguishing it from the teaching of reading. Thus, our first rule for technological fixes is:

I. The technology must largely embody the cause-effect relationship connecting problem to solution.

A key point, well-illustrated by have been made in disease reduction through vaccination efforts, for example. But the story for literacy is different. In the United States, nearly a half century of research, application of new technologies and development of new methods and policies has failed to translate into improved reading abilities for the nation's children ${ }^{1}$.

Although vaccinating children and teaching them to read may seem so different as to make them incommensurable, they are similar in several important respects. Both are carried out by trained professionals in a controlled environment using the standard tools of their respective trades. Notably, each has been, and continues to be, the subject of considerable research. But the reasons why progress has been so uneven point to three simple rules for anticipating when more research and development (R\&D) could help to yield rapid social progress. In a world of limited resources, the trick is distinguishing problems amenable to technological fixes from those that are not. Our rules provide guidance in making this distinction, be it for education, disease prevention or even climate change.

\section{Square peg, round hole}

Both vaccinating and teaching involve skilfully produced artefacts. But unlike vaccines, the textbooks and software used in education do not embody the essence of what needs to be done. That is, they don't provide the basic 'go' of teaching and learning. That depends on the skills of teachers and on the attributes of classrooms and students. Most importantly, the effectiveness of a vaccine is largely independent of who gives or receives it, and of the setting in which it is given. A health-care practitioner (unlike a teacher) doesn't usually have to figure out what will work on a case-by-case basis - no matter if the child is rich or poor, if he or she speaks English or Mandarin. The vaccine captures the basic go in a technological vaccines, is that a technological fix needs to be successful within the context of a complex sociotechnical system that is difficult to understand, let alone manage. The health-care system in the United States, for example, is notoriously dysfunctional, inequitable and resistant to successful reform, yet most children manage to get vaccinated. Vaccines offer a clear advantage over other approaches to protecting children from certain infectious diseases. This advantage dampens disagreement about alternative approaches. Such clarity allows policy and operational coordination to emerge among diverse actors and institutions, ranging from doctors and parents to school districts, insurance companies, vaccine manufacturers and regulatory bodies.
Yet in the case of reading, an equally diverse set of actors and institutions remains in a continual state of conflict regarding how best to improve performance, and the education system itself is often blamed for the lack of improvement. Our point is that performance could improve despite system complexities if a broadly effective method of teaching reading were developed. The problem is that decades of effort have not led to such a method ${ }^{2-4}$. Different approaches to improved teaching remain strongly context dependent, and no particular approach confers an obvious advantage over others in all circumstances. Adherents of every approach have citable evidence to back their position, reinforcing their sense that 'the system' is the problem. These observations lead to our second rule:

II. The effects of the technological fix must be assessable using relatively unambiguous or uncontroversial criteria.

From their earliest use, vaccines provoked opposition on moral and practical grounds, a trend that continues today. But opposition has not stemmed the long-term advance of vaccines. This is in part because their effectiveness is hard to argue against and because continual improvement has tended to answer objections about efficacy and risk. The situation stands in stark contrast to the teaching of reading, for which no particular method or theory has been able to achieve long-term or widespread dominance and for which compelling evidence of improved efficacy even over timescales of a century is lacking.

For vaccination, the standardized core, the vaccine - first developed more than two centuries ago not through basic research but through empiricism guided by folk wisdom - remains the fulcrum on which cumulative learning and improved practice can be leveraged. This makes it possible to experiment with confidence that what is learned can be applied to standard practice and used by a range of practitioners, and leads to our third rule:

III. Research and development is most likely to contribute decisively to solving a social problem when it focuses on 
improving a standardized technical core that already exists.

Scientific understanding related to a standardized core is much easier to apply than science aimed at elucidating the theoretical foundations, causes or dynamics of a problem. When knowledge is not largely embodied in an effective technology, but must instead be applied to practice through, say, training, institutional incentives, organizational structures or public policies, the difficulty of improving outcomes is greatly amplified. Now the task involves moulding, coordinating and governing the activities of practitioners, who themselves must acquire judgement and skill that may not be easily translatable from one context to another. Interpreting the results of management or policy innovations is difficult because of the many variables involved, few of which are directly related to the actual technology deployment. When the results of applying knowledge to practice are uncertain, the value of the new knowledge itself becomes subject to controversy.

\section{The limits of technology}

In the absence of an existing standardized core, therefore, $\mathrm{R} \& \mathrm{D}$ programmes aimed at solving particular social problems should neither be expected to succeed, nor be advertised as having much promise of succeeding, at least in the short and medium term. They should be understood and described as aiming at the creation of fundamental knowledge and the exploration of new approaches, with success possible only over the long term, and with a significant chance of failure.

We are not, of course, arguing against working hard to address social problems that are not amenable to technological fixes, but we are saying that $R \& D$ is unlikely to be the main source of short- or medium-term progress. So long as the teaching of reading, for example, cannot be condensed into an easily deployed technology or tightly specified routine, improvement will mostly come through context-dependent trial and error at the level of public policy and organizational management. This is more a process of effective politics than effective innovation, and typically progress will be slow, hard-fought and uneven. On the other hand, when a standardized technological core relevant to a particular problem is available, appropriate $R \& D$ investments have the potential to stimulate rapid progress.

How might these insights help guide innovation policies today? To illustrate the implications of our rules for decision-makers, we turn briefly to climate change.

The global energy system that lies at the heart of the climate-change problem is probably more complex and resistant to successful reform than the health-care and education systems discussed above. Despite enormous scientific, political and diplomatic efforts over the past two decades, no progress on reducing global greenhouse-gas emissions has been made ${ }^{5,6}$. In the absence of technological fixes, progress towards significant reductions of greenhouse-gas emissions will remain frustratingly slow, uneven and inconclusive.

What are the prospects for a technological fix? In principle, stabilizing atmospheric carbon dioxide concentrations at levels deemed acceptable by climate experts can be achieved through radically reduced emissions or through direct removal of $\mathrm{CO}_{2}$ from the atmosphere. Most discussion and effort focuses on the former. The suite of promising possibilities for reducing emissions - from nuclear fission, to photovoltaics, to on-site carbon capture and storage - offers attractive targets for R\&D investments ${ }^{7}$ consistent with Rule III: existing technological capacities can leverage continued improvement. Nevertheless, successful transition to a low-emissions energy system requires effective management across all sectors of society and all uses of $\mathrm{CO}_{2}$-emitting technologies. Within this system, no particular technology fully encompasses the go of the process - eliminating $\mathrm{CO}_{2}$ in the atmosphere - just as no particular teaching technology encompasses the go of teaching children to read. Rule I is violated.

Moreover, because emissions-reducing technologies will compete with existing energy technologies supported by entrenched interests, and because there will be competition between the emerging technologies, we can expect ongoing technical and political debates about efficacy of specific technologies, as seen for biofuels today - a violation of Rule II. System-wide progress is therefore likely to be buffered by political processes similar to the ones that frustrate progress now.

In contrast, direct removal of $\mathrm{CO}_{2}$ from the atmosphere - air capture ${ }^{8}$ - satisfies the rules for technological fixes. Most importantly, air capture embodies the essential cause-effect relations - the basic go - of the climatechange problem, by acting directly to reduce $\mathrm{CO}_{2}$ concentrations, independent of the complexities of the global energy system (Rule I). There is a criterion of effectiveness that can be directly and unambiguously assessed: the amount of $\mathrm{CO}_{2}$ removed (Rule II). And although air-capture technologies have been remarkably neglected in both $\mathrm{R} \& \mathrm{D}$ and policy discussions, they nevertheless seem technically feasible (Rule III) ${ }^{9-11}$.

Our rules do not allow us to predict if aircapture technologies will in fact help stabilize greenhouse-gas concentrations. Certainly these technologies face technical, political and economic obstacles. Our rules do, however, allow us to strongly predict that stabilization is unlikely to be achieved, except in the very long term, without something like air capture. Such technologies should therefore receive much greater attention in energy innovation portfolios.

The climate-change example illustrates an important final point: technological fixes do not offer a path to moral absolution, but to technical resolution. Indeed, one of the key elements of a successful technological fix is that it helps to solve the problem while allowing people to maintain the diversity of values and interests that impede other paths to effective action. Recognizing when such opportunities for rapid progress are available should be a central part of innovation policy, and should guide investment choices.

Daniel Sarewitz is co-director of the Consortium for Science, Policy \& Outcomes, and Professor of Science and Society, at Arizona State University, Tempe, Arizona 85287, USA.

e-mail: daniel.sarewitz@asu.edu

Richard Nelson is George Blumenthal Professor of International and Public Affairs Emeritus at Columbia University, New York 10027, USA, and visiting professor at the University of Manchester Business School.

e-mail:rrn2@columbia.edu

1. http://nces.ed.gov/nationsreportcard/Itt/results2004

2. Donovan, M. S., Wigdor, A. K. \& Snow, C. E. (eds) Strategic Education Research Partnership (National Academies Press, 2003).

3. Foray, D. \& Hargreaves, D. Lond. Rev. Edu. 1, 7-19 (2003).

4. Murnane, R. \& Nelson, R. Economics of Innovation and New Technology 16, 307-327 (2007)

5. Prins, G. \& Rayner, S. Nature 449, 973-975 (2007).

6. Sarewitz, D. \& Pielke Jr, R. in Controversies in Science and Technology, vol. 2: From Chromosomes to the Cosmos (eds Kleinman, D. L. \& Handelsman, J.) 329-351 (Mary Ann Liebert, 2008).

7. Hoffert, M. I. et al. Science $\mathbf{2 9 8}, \mathbf{9 8 1 - 9 8 6}$ (2002).

8. Keith, D. W., Ha-Duong, M. \& Stolaroff, J. K. Climatic Change 74, 17-45 (2006)

9. Baciocchi, R., Storti, G. \& Mazzotti, M. Chem. Eng. Process. 45, 1047-1058 (2006)

10. Lackner, K. S. Science 300, 1677-1678 (2003).

11. Stolaroff, J. Capturing $\mathrm{CO}_{2}$ from Ambient Air: A Feasibility Assessment (PhD Dissertation, Carnegie Mellon University, 2006).

To comment on this article and others in our innovation series, visit http://tinyurl.com/5uolx 2 .

\section{Correction}

The Commentary 'Towards responsible use of cognitive-enhancing drugs by the healthy' (Nature 456, 702-705; 2008) described views derived from the report Beyond Therapy as solely those of Leon Kass. In fact, the work in question was by the President's Council on Bioethics, which at that time Kass chaired. 